\title{
Searching for an optimal switched reluctance motor design
}

\author{
WieStaW JażdżyŃSKi ${ }^{1}$, Michal MAJChrowicz ${ }^{2}$ \\ ${ }^{1}$ AGH University of Science and Technology \\ Al. Mickiewicza 30, 30-059, Kraków, Poland \\ e-mail:wjaz@agh.edu.pl \\ ${ }^{2}$ Nidec Motors \& Actuators (Poland), Sp. z o.o. \\ ul. Skarbowa 36, Niepołomice 32-005, Poland \\ e-mail: michal.majchrowicz@nidec-ma.com
}

(Received: 09.09.2014, revised: 06.10.2014)

\begin{abstract}
An approach helpful when developing an optimized construction of a 6/4 type switched reluctance motor (SRM) is described in the paper. The analytical modeling procedure, based on the reluctance network method and analytical solution of an ordinary differential equation, enables applying a gradient optimization routine and better control of optimization process. The model allows for estimation of the efficiency, torque, and acoustic noise of the motor taking into account the magnetic non-linearity and the control algorithm to keep a constant input power. A bicriterial optimization routine has been applied to find optimal constructions. Eleven geometric and winding parameters are supposed to be the optimization quantities. Analyzed constructions - the initial one and the optimized ones, were validated by means of FEM calculations. The proposed approach can be employed in designing the SRM to be a drive motor in an electrical vehicle, at least as a first attempt.
\end{abstract}

Key words: switched reluctance motor, optimization, analytical model

\section{Introduction}

A motor designed for an electric vehicle drive should meet requirements, which are difficult to satisfy simultaneously. In the case of a switched reluctance motor (SRM) they can be: a high mean value of the electromagnetic torque, a low level of torque ripples, high efficiency, low acoustic noise and low motor cost or mass. There are many strategies to reach this goal. Usually the motor construction is optimized first $[1,2]$, and next the best control algorithm is searched for [3]. Many papers devoted to optimization of the SRM are based on FEM models with auxiliary lumped parameter parts defined by means of analytical formulae like in $[2,4]$, [5]. In optimization, evolutionary algorithms [6-8] are used more often than other methods, i.e. gradient optimization routines [9]. The presented approach is a summary of the research stage started by the authors a few years ago [10-15]. 
An initial construction in optimization is a 6/4 type SRM described in [16]. Experimental results presented there we have considered as a credible relevance in the model verification process. A multicriterial optimization approach is employed in this paper when searching for the best design. Criterial functions have been selected to be the mean value of the electromagnetic torque, the relation mean to max value of the electromagnetic torque, efficiency, magnetic noise level and motor mass. The main part of a synthesis program is the analytical model described and verified in [15], which enables determining all quantities necessary to define criterial functions and feasible region in optimization, in particular the electromagnetic torque parameters, the power loss components, and the magnetic radial force, for a constant angular velocity. The magnetic non-linearity is represented by means of a $\mathrm{B}-\mathrm{H}$ characteristic of the M400-50A electrical sheet. Mutual inductances were neglected, but consequences of this assumption are discussed in [15].

The paper is focused on optimization problems. A lumped-parameter model of the SRM is applied, but optimization solutions have been verified by means of FEM analysis.

A common approach in the process of developing an electric machine consists of several successive cycles $\{$ research $\Rightarrow$ prototype $\Rightarrow$ experimental verification . A wrong idea can usually be rejected after the first one, and at least two cycles are required to reach satisfactory results, but there are cases with more cycles, which after a dozen years offer spectacular results [17]. This paper relates to the "research" stage, with verification by means of FEM calculation results. Experimental data in $[16,18]$ are also taken into account in this process.

\section{Analytical model of the SRM}

\section{A. Description of the model}

All important motor quantities, for instance the phase current, electromagnetic torque, radial force operating on stator pole, and a flux density in the stator pole, are determined in the model as time functions dependent on motor parameters and operation conditions, with accuracy required for gradient optimization routines. A constant motor velocity and one pulse mode operation of the inverter is assumed in computation. Some preliminary simulation results had been published in [13] and [15], and more detailed information can be found in [14].

The input quantities in the synthesis program are: geometrical dimensions $r_{\mathrm{o}}, r_{\mathrm{sy}}, r_{\mathrm{sp}}, r_{\mathrm{ry}}$, $r_{\mathrm{sh}}, b_{\mathrm{sp}}, b_{\mathrm{rp}}, g$ (Fig. 1), stack length $l$, number of turns in the phase winding $T_{\mathrm{ph}}$, wire diameter, DC voltage $E$ supplying phase circuit, rotor angular velocity $\omega=n^{*} \pi / 30$, and assumed input active power $P_{\text {in }}$.

In the first step of calculations, based on the reluctance network method described in [19], [10] the phase winding inductance $L(i, \varphi)$ is determined as a function of phase current $i$ and angular rotor position $\varphi$. In the next step, basing upon the inductance $L(i, \varphi)$, a function of a flux linkage $\Psi(i, \varphi)$ and its both partial derivatives are calculated.

The phase winding resistance $R$ is determined basing on dimensions of the stator pole and winding parameters. In the paper it comprises an additional resistance representing the sup- 
plying wires, the converter elements, and the internal resistance of the constant voltage source as well.

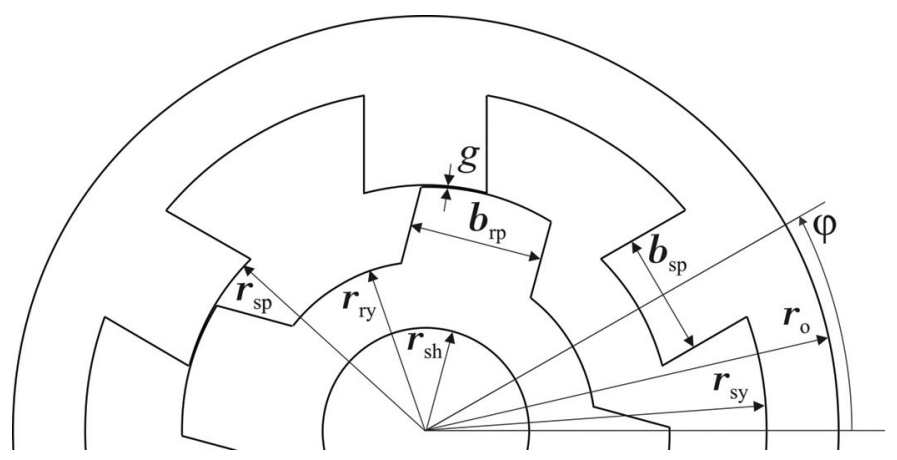

Fig. 1. Geometrical parameters of the magnetic core

The torque function $T_{\mathrm{el}}$ is calculated from a magnetic co-energy function:

$$
T_{\mathrm{el}}\left(\mathrm{i}_{\mathrm{x}}, \varphi\right)=\frac{\partial}{\partial \varphi} \int_{0}^{i_{\mathrm{x}}} L(\mathrm{i}, \varphi) i \mathrm{~d} i
$$

The phase current $i(\mathrm{t})$, assumed to be a linear spline, is calculated as an analytical solution of the following non-linear differential equation:

$$
u=R i+\frac{\partial \Psi(\mathrm{i}, \varphi)}{\partial i} \frac{\mathrm{d} i}{\mathrm{~d} t}+\omega \frac{\partial \Psi(\mathrm{i}, \varphi)}{\partial \varphi},
$$

where: $u$ - phase voltage, $i$ - phase current, $t$ - time, $\omega$ - rotor angular velocity, $\varphi$ - angular rotor position, $R$ - phase resistance, $\Psi$ - flux linkage.

In the paper, Equation (2) is solved for a constant angular velocity $\omega=n^{*} \pi / 30$, for $n=3500 \mathrm{rpm}$, and a phase voltage function $u(t)$ defined below [15]:

$$
u=\left\{\begin{array}{c}
i(0)=0 ; i \geq 0 ; \\
E, \text { for } \varphi \in\left[0, \varphi_{\mathrm{off}}\right] \text { and } i \geq 0 \\
-E, \text { for } \varphi \in\left(\varphi_{\mathrm{off}}, \frac{2 \pi}{N_{\mathrm{r}}}\right] \text { and } i>0 \\
0, \text { for } \varphi \in\left(\varphi_{\mathrm{off}}, \frac{2 \pi}{N_{\mathrm{r}}}\right] \text { and } i=0 .
\end{array}\right.
$$

The Equation (2) is solved for one phase and a revolution angle equal to one rotor pole pitch. The voltage $E$ value in the optimization approach is $160 \mathrm{~V}$. The $\varphi_{\text {off }}$ angle is obtained as 
a solution of non-linear algebraic equation in each optimization iteration, to keep the required reference value of the motor input active power $P_{\text {in }}=35 \mathrm{~kW}$ at the assumed rotor velocity $3500 \mathrm{rpm}$. Poles of one phase are connected in parallel. These above assumptions concern all optimization calculations performed in this paper.

In the next step, the maximal values of the flux density in the main magnetic core parts, time functions of a torque and a magnetic radial force, are calculated. Detailed calculation procedure can be found in [15].

\section{B. Model validation}

The quality of optimization results depends strongly on an accuracy of the model. Necessary validation calculations have been performed by means of a $2 \mathrm{D}$ finite element method. Results can be found in [13-15]. A comparison with experiments performed for almost the same switched reluctance machine [16, 18], see Figure 2 [15], seems to be acceptable, despite a level of uncertainty caused by the model assumptions, in particular:

- no PWM, only one-pulse mode supplying voltage,

- a constant turn-on angle $\varphi_{\text {on }}=0^{\circ}$,

- the inverter consists of ideal elements,

- very likely an electrical steel has another properties.
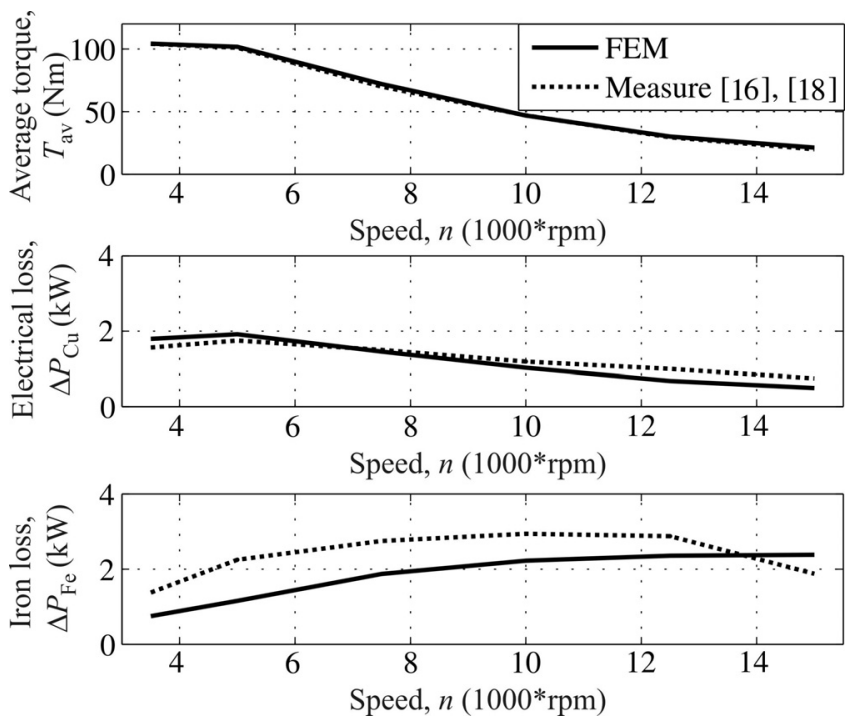

Fig. 2. The comparison of average torque, electrical loss and iron loss components for FEM model and the real machine $[16,18]$

\section{Quantities used in optimization}

The following quantities used in the synthesis program to define criterial functions and a feasible region are derived in the model: the average value $T_{\text {av }}$ of total electrical torque, the ratio $T_{\text {av2max }}$ of the $T_{\text {av }}$ to the max $T_{\text {el }}$ (the torque ripples quantity), efficiency $\eta$, acoustic noise 
level $L_{\mathrm{wA}}$ (a magnetic component) and total mass $m_{\mathrm{m}}$ (or the cost of materials). Detailed calculating procedures for these quantities can be found in [15].

An accuracy of efficiency calculation depends on accuracy of the iron loss estimator, what is troublesome due to a complex shape of the flux density functions in different core parts. In the paper the iron loss is estimated on assumption that the functions of magnetic flux density in motor core parts can be approximated by means of a linear spline [15].

The acoustic noise prediction is based on an analytical model described in [4, 20, 21]. It comprises the following analyze components: determining a time function of magnetic radial force, a frequency domain analysis of the radial force, a modal analysis of the stator, determining an amplitude of dynamic deflection of the stator, and a sound power radiated by the motor [15]. The modes $m=0,2,4$ representing the majority of sound power for $6 / 4$ type SRM [22] are considered in the model.

\section{Optimization}

A few examples of optimization calculations have been performed to test the analytical model and to improve the properties of the analyzed construction. In the first stage a few so called ideal solutions for selected criterial functions have been found, to have a basic knowledge what are the extreme performances of this construction. It was an opportunity to modify the model and feasible region. Next a bicriterial approach has been performed with criterial functions arising from the analysis at the first stage.

\section{A. Limitations of the model}

The following simplifications have been assumed:

- the winding temperature was assumed to be constant $\tau_{\mathrm{Cu}}=180^{\circ} \mathrm{C}$, a value close to that given in [16],

- the flux density values in the yokes are calculated basing upon the superposition rule,

- the analytical model neglects mutual inductances.

The above simplifications influence motor properties and it must be taken into account during optimization. In the paper it was obtained by means of:

- upper limits imposed on the maximal values of the flux density in the middle of motor poles and yokes $\leq 1,65 \mathrm{~T}$,

- an upper limit value of the turn off angle $\varphi_{\text {off }} \leq 360 / N_{\mathrm{ph}} / N_{\mathrm{r}}=30^{\circ}$,

- a reduction of the supplying voltage from $215 \mathrm{~V}$ to $160 \mathrm{~V}$ at $3500 \mathrm{obr} / \mathrm{min}$ (only so called one pulse mode operation of the inverter was assumed).

\section{B. Optimization variables}

The optimization variables vector $\mathbf{x}$ is defined in Table 1 (first column). It comprises geometrical parameters defined on Figure 1, stack length $l$, and parameters of the winding: the number of turns per phase $T_{\mathrm{ph}}$ and the wire cross section diameter $d_{1 \mathrm{~T}}$.

All dimensions with except $T_{\mathrm{ph}}$ are in (mm). The initial construction is denoted $\mathbf{x}_{0}$. 


\section{Criterial functions}

Almost all quantities describing motor properties can be selected as criterial functions. In the paper, the basic set of these functions was assumed to be $\left\{\eta, T_{\mathrm{av}}, T_{\mathrm{av} 2 \max }, L_{\mathrm{wA}}, m_{\mathrm{m}}\right\}$. If at least two of them are not cooperative, they can be employed in a multicriterial approach.

\section{Feasible region}

In general, the task of constraint functions is to keep a solution inside a feasible region, where all kinds of quantitative requirements imposed on it should be satisfied.

Only inequality (linear and non-linear) constraints have been used in the definitions of optimization problems in the paper. The equality constraint $P_{\text {in }}\left(\varphi_{\text {off }}\right)=35 \mathrm{~kW}$ was solved in the model directly. Some constraints arise from the calculation procedure in the model, see Appendix.

The constraints in Tables 6 and 7 in the Appendix define the basic feasible region $X$, which can be extended, to meet optimization problem requirements. Due to a level of uncertainty about technology, they have been assumed basing upon a general knowledge and a common sense.

\section{E. Optimization results}

\section{Stage I. Ideal solutions}

A few scalar optimization problems (4)-(7) have been solved to find a set of non-cooperative criterial functions in a multicriterial approach. Some results of these calculations are in Tables 1, 2 and Figures 3-6.

Problem P1.

$$
\max _{\mathbf{x}} T_{\text {av2max }} \mid \mathbf{x} \in X, \quad T_{\text {av }} \geq T_{\text {av0 }}, \quad m_{\mathrm{m}} \leq m_{\mathrm{m}_{0}}
$$

Problem P2.

$$
\max _{\mathbf{x}} \eta \mid \mathbf{x} \in X, \quad T_{\text {av2max }} \geq T_{\text {av2max } 0}, T_{\mathrm{av}} \geq T_{\text {av0 }}, m_{\mathrm{m}} \leq m_{\mathrm{m} 0}, L_{\mathrm{wA}} \leq L_{\mathrm{wA} 0} .
$$

Problem P3.

$$
\max _{\mathbf{x}} y_{\mathrm{s}} \mid \mathbf{x} \in X, \quad T_{\text {av2max }} \geq T_{\text {av2max } 0}, \quad T_{\text {av }} \geq T_{\text {av0 }}, \quad \eta \geq \eta_{0} .
$$

Problem P4.

$$
\min _{\mathbf{x}} L_{\mathrm{wA}} \mid \mathbf{x} \in X, \quad T_{\operatorname{av} 2 \max } \geq T_{\mathrm{av} 2 \max 0}, \quad T_{\mathrm{av}} \geq T_{\mathrm{av} 0}, \quad \eta \geq \eta_{0} .
$$

The quantity $\mathbf{x}$ is a vector of optimization variables, and $X$ - the basic feasible region. The index " 0 " corresponds to the initial construction.

The goal of both Problem P3 and P4 is a noise level minimization. Problem P3 represents an idea to simplify calculations by searching for a maximum value of the stator yoke thickness instead of solving Problem P4. A comparison of the data in Table 2 shows that a similar result 
for a criterial function $L_{w A}$ has been achieved in both cases, although corresponding design quantities $\mathbf{x}$ in Table 1 differ.

One can see in Table 2 that in all Problems the qualitative change of criterial functions is the same in both models, analytical (AM) and finite element (FEM). It can be considered as a kind of a successful verification of the analytical model.
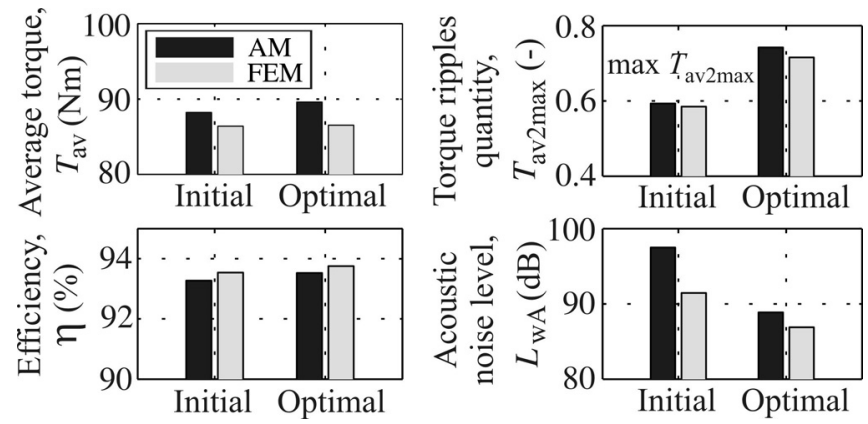

Fig. 3. Problem P1. Minimization of the torque ripples;

AM - analytical model, FEM - finite element simulations
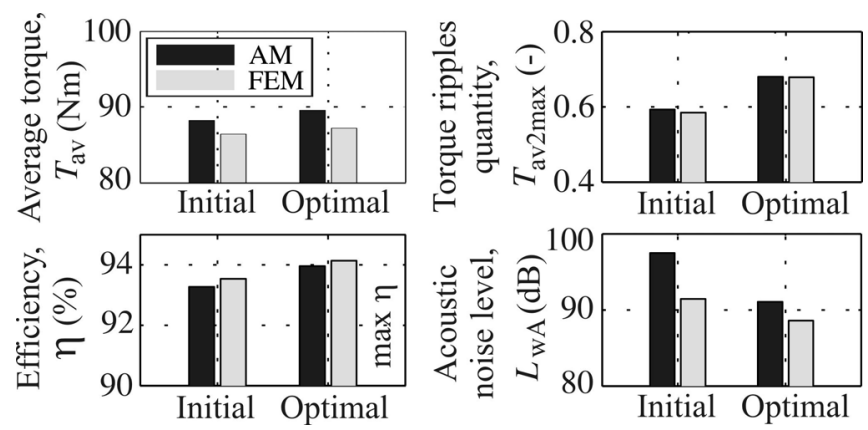

Fig. 4. Problem P2. Maximization of the efficiency; AM - analytical model, FEM - finite element simulations
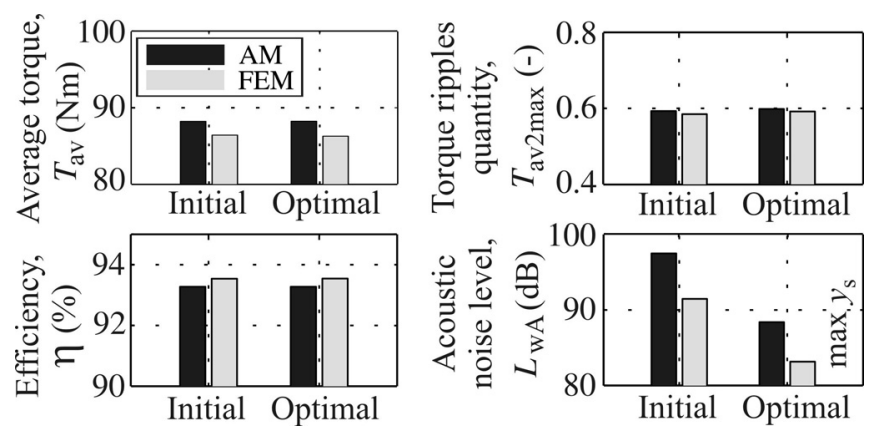

Fig. 5. Problem P3. Maximization of the stator yoke; $\mathrm{AM}$ - analytical model, FEM - finite element simulations 

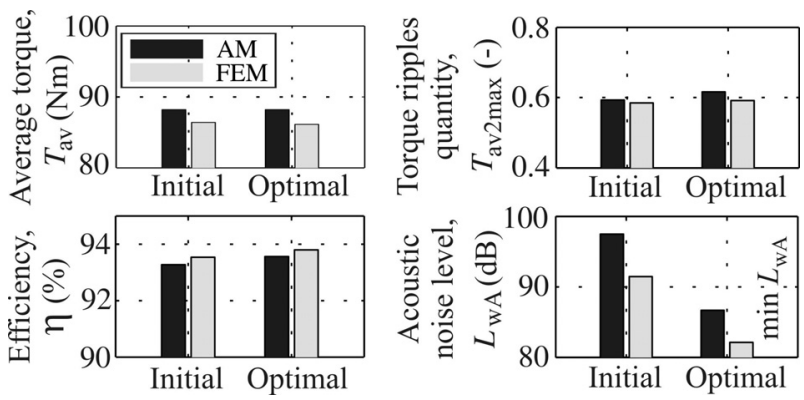

Fig. 6. Problem P4. Minimization of the noise level; AM - analytical model, FEM - finite element simulations

Table 1. Ideal solutions - variables

\begin{tabular}{c|c|c|c|c|c}
\hline \multirow{2}{*}{ Parameter $\boldsymbol{x}_{\boldsymbol{i}}$} & Starting value & \multicolumn{4}{|c}{ Relative change $\left(\boldsymbol{x}_{\boldsymbol{i}} / \boldsymbol{x}_{\boldsymbol{i} \mathbf{0}}-\mathbf{1}\right) * \mathbf{1 0 0 \%}$ for problem } \\
\cline { 3 - 6 } & $\boldsymbol{x}_{\boldsymbol{i} \mathbf{0}}(\mathbf{m m})$ & $\mathrm{P} 1$ & $\mathrm{P} 2$ & $\mathrm{P} 3$ & $\mathrm{P} 4$ \\
\hline$x_{1}=b_{\mathrm{sp}}$ & 31.55 & 5.6133 & -1.9461 & -0.0444 & -8.4216 \\
\hline$x_{2}=r_{\mathrm{o}}$ & 106 & 0.0000 & 0.0000 & 0.0000 & 0.0000 \\
\hline$x_{3}=r_{\mathrm{sy}}$ & 87.5 & 0.4823 & 2.7349 & -0.8834 & 0.4320 \\
\hline$x_{4}=r_{\mathrm{sp}}$ & 63 & -6.1556 & -2.8524 & -0.6683 & -6.8429 \\
\hline$x_{5}=b_{\mathrm{rp}}$ & 34.51 & -3.4454 & -3.2367 & -0.1130 & -16.7256 \\
\hline$x_{6}=r_{\mathrm{sh}}$ & 26.5 & 0.0000 & 0.0000 & 0.2340 & 10.3698 \\
\hline$x_{7}=r_{\mathrm{ry}}$ & 43.5 & -0.7793 & 3.0552 & 0.3494 & 0.4483 \\
\hline$x_{8}=l$ & 215 & -1.2233 & -0.9302 & -1.3953 & -0.8047 \\
\hline$x_{9}=g$ & 0.4 & 0.0000 & 0.0000 & 0.0000 & 0.0000 \\
\hline$x_{10}=T_{\mathrm{ph}}$ (turns) & 38 & -1.4632 & 6.0553 & 0.2553 & 8.7026 \\
\hline$x_{11}=d_{1 \mathrm{~T}}$ & 3.669 & 2.1559 & 8.0294 & -0.4252 & 7.2309 \\
\hline \multicolumn{5}{|c|}{}
\end{tabular}

AM - analytical model, FEM - finite element method

A relatively little variation of the efficiency $\eta$ in Table 2 arises from severe restrictions imposed on the motor construction, and also from the fact, that the motor design, assumed in the paper to be initial one in optimization, corresponds to a final, very likely optimized construction in [16].

\section{Stage II. Bicriterial optimization}

Any two criterial functions from the set $\left\{T_{\mathrm{av} 2 \max }, \eta, L_{\mathrm{wA}}\right\}$ can be employed in the bicriterial approach, see Table 2, Figures 3, 4, and 6.

Additional calculations show that a similar conclusion holds if the average electromagnetic torque $T_{\mathrm{av}}$ is an element of this set. Finally we have decided to solve the following bicriterial problem.

Problem P5.

$$
\min _{\mathbf{x}} L_{\mathrm{wA}}, \max _{\mathbf{x}} T_{\mathrm{av}} \mid \mathbf{x} \in X
$$


Table 2. Ideal solutions - criterial functions values change

\begin{tabular}{|c|c|c|c|c|c|}
\hline \multirow{2}{*}{ Criterial functions $f_{i}$} & \multirow{2}{*}{ Starting point $f_{i 0}$} & \multicolumn{4}{|c|}{ Relative change $\left(f_{i} / f_{i 0}-1\right) * 100 \%$ for problem } \\
\hline & & P1 & $\mathrm{P} 2$ & P3 & P4 \\
\hline$T_{\text {av,AM}},(\mathrm{Nm})$ & 88.204 & 1.5725 & 1.5237 & 0.0306 & 0.0000 \\
\hline$T_{\text {av,FEM }},(\mathrm{Nm})$ & 86.393 & 0.1470 & 0.9029 & -0.1563 & -0.3183 \\
\hline$T_{\text {av2max,AM }},(-)$ & 0.5931 & 25.122 & 14.686 & 0.9611 & 3.9285 \\
\hline$T_{\text {av2max,FEM }},(-)$ & 0.5848 & 22.315 & 16.057 & 1.2483 & 1.1799 \\
\hline$\eta_{, \mathrm{AM}},(\%)$ & 93.273 & 0.2702 & 0.7344 & 0.0000 & 0.3120 \\
\hline$\eta_{\text {,FEM }},(\%)$ & 93.539 & 0.2320 & 0.6414 & 0.0000 & 0.2748 \\
\hline$L_{\mathrm{wA}, \mathrm{AM}},(\mathrm{dB})$ & 97.478 & -8.8297 & -6.5730 & -9.3324 & -11.086 \\
\hline$L_{\mathrm{wA}, \mathrm{FEM}},(\mathrm{dB})$ & 91.453 & -4.9949 & -3.1098 & -9.0724 & -10.201 \\
\hline$m_{\mathrm{m}},(\mathrm{kg})$ & 46.217 & -0.0022 & 0.0000 & -0.3981 & 0.4825 \\
\hline
\end{tabular}

AM - analytical model, FEM - finite element method

Selection of the best compromise solution

Some obtained results are presented on Figures 7 and 8. Figure 7 represents an estimate of the compromise solutions set in the objective space.

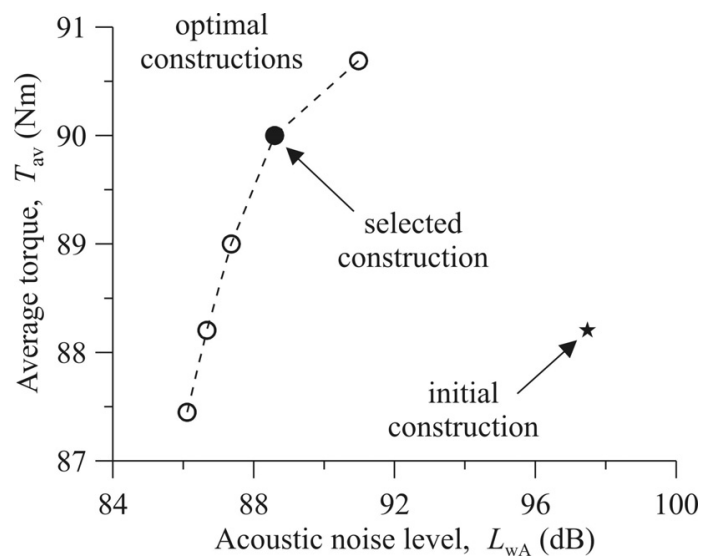

Fig. 7. Problem P5. Bicriterial approach, compromise solution set in the objective space

Additional criteria must be applied to decide, which element of the compromise solution set is the best. The values of criterial functions of $\eta, T_{\text {av2max }}$ and $m_{\mathrm{m}}$, can be helpful in this task.

A solution denoted as "selected construction" on Figure 7 offers a considerable improvement for both criterial functions $T_{\mathrm{av}}$ and $L_{\mathrm{wA}}$ compared to the "initial construction", and can be considered as the best. The data describing this construction are given in Table 3 and Figure 8 . One can see that all criterial function values have been improved.

Inequality constraints in optimization can be presented in a form $g_{j}(\mathbf{x}) \leq G_{j}$, where $g_{j}$ is variable, and $G_{j}$ - constant part of the constraint function. If $G_{j}$ is altered to a new value 
$G_{j}^{\text {new }}=G_{j}+\Delta G_{j}$, and in both cases the $j t h$ constraint remains active in the optimal point, than a new optimal value of the objective function $F_{\text {opt }}^{\text {new }}$ can be estimated from a formula:

$$
F_{\mathrm{opt}}^{\mathrm{new}} \cong F_{\mathrm{opt}}+\Delta F_{\mathrm{opt}} \cong F_{\mathrm{opt}}-\sum_{j} \lambda_{j} \Delta G_{j}
$$
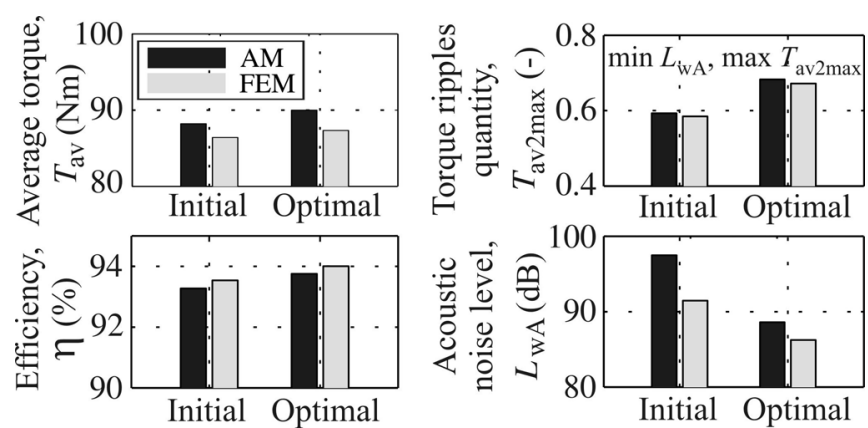

Fig. 8. Problem P5. Motor quantities for "selected construction", AM - analytical model, FEM - finite element simulations

Table 3. Problem P5. The selected construction. A change in $(\%)=\left(X_{I} / X_{I 0}-1\right) * 100 \%$, and $\left(F_{I} / F_{I 0}-1\right) * 100 \%$

\begin{tabular}{c|c|c|c|c|c}
\hline \multirow{2}{*}{$x_{i}$} & \multicolumn{2}{|c|}{ Parameter } & \multirow{2}{*}{$f_{i}$} & \multicolumn{2}{c}{ Function } \\
\cline { 2 - 3 } \cline { 5 - 6 } & value $(\mathrm{mm})$ & a change $(\%)$ & & value & a change $(\%)$ \\
\hline$b_{\mathrm{sp}}$ & 31.048 & -1.5911 & $T_{\mathrm{av}, \mathrm{AM}}(\mathrm{Nm})$ & 90.000 & 2.0362 \\
\hline$r_{\mathrm{o}}$ & 106 & 0.0000 & $T_{\text {av }, \mathrm{FEM}}(\mathrm{Nm})$ & 87.316 & 1.0684 \\
\hline$r_{\mathrm{sy}}$ & 88.249 & 0.8560 & $T_{\text {av2max }, \mathrm{AM}}(-)$ & 0.6829 & 15.141 \\
\hline$r_{\mathrm{sp}}$ & 62.296 & -1.1175 & $T_{\mathrm{av} 2 \max , \mathrm{FEM}}(-)$ & 0.6720 & 14.911 \\
\hline$b_{\mathrm{rp}}$ & 35.438 & 2.6891 & $\eta_{\text {AM }}(\%)$ & 93.750 & 0.5114 \\
\hline$r_{\mathrm{sh}}$ & 28.35 & 6.9811 & $\eta_{\text {FEM }}(\%)$ & 94.002 & 0.4950 \\
\hline$r_{\mathrm{ry}}$ & 43.874 & 0.8598 & $L_{\mathrm{wA}, \mathrm{AM}}(\mathrm{dB})$ & 88.597 & -9.1108 \\
\hline$l$ & 213.67 & -0.6186 & $L_{\mathrm{wA}, \mathrm{FEM}}(\mathrm{dB})$ & 86.238 & -5.7024 \\
\hline$g$ & 0.4 & 0.0000 & $m_{\mathrm{m}}(\mathrm{kg})$ & 46.346 & 0.2791 \\
\hline$T_{\mathrm{ph}}(\mathrm{turns})$ & 39.754 & 4.6158 & & & \\
\hline$d_{1 \mathrm{~T}}$ & 3.7594 & 2.4639 & & & \\
\hline
\end{tabular}

AM - analytical model, FEM - finite element method

without solving the problem with a modified feasible region. The quantities $\lambda_{j}$ are Lagrange multipliers obtained from a solution of the original problem. The relationship (9) is of practical meaning. For instance, if a question is how the efficiency $\eta$ of the motor can be increased through a change $\Delta m_{\mathrm{m}}$ of its mass $m_{\mathrm{m}}$ in Problem P2, than the definitions $F=2-\eta / \eta_{0}$, $g_{j}=\left(m_{\mathrm{m}} / m_{\mathrm{m} 0}-1\right)$ used in this Problem, and obtained value $\lambda_{j}=0.0315$ in Table 4 give $\Delta \eta_{\text {opt }} / \eta_{0} \cong 0.0315 \cdot \Delta m_{\mathrm{m}, \mathrm{opt}} / m_{\mathrm{m} 0}$. 
Table 4. Active constraints

\begin{tabular}{c|c|c|c|c}
\hline \multirow{2}{*}{$\begin{array}{c}\text { Constraint No } \\
\text { (Appendix C) }\end{array}$} & \multicolumn{4}{|c}{ Lagrange multipliers $\boldsymbol{\lambda}_{\boldsymbol{j}}$ for Problem } \\
\cline { 2 - 5 } & $\mathrm{P} 1$ & $\mathrm{P} 2$ & $\mathrm{P} 4$ & $\begin{array}{c}\mathrm{P} 5 \\
\text { (“selected constr.") }\end{array}$ \\
\hline 1 & -578.95 & -34.759 & -740.31 & -77.474 \\
\hline 7 & 0.0880 & 0.0114 & 0 & 0 \\
\hline 10 & 0 & 0 & 0.0301 & 0.0686 \\
\hline 14 & 0.187 & 0.0326 & 0 & 0.441 \\
\hline 27 & 0 & 0 & 5.132 & 0 \\
\hline 30 & 0 & 0 & 2.769 & 0 \\
\hline 33 & 0 & 0 & 0 & 0 \\
\hline 34 & 0.395 & 0 & 0 & 0.385 \\
\hline 36 & 0 & 0 & 0.334 & 0 \\
\hline 39 & 1.718 & 0 & 0 & 0 \\
\hline 41 & 0 & 0.0263 & 0.0404 & 0.0789 \\
\hline 42 & 0.147 & 0.00637 & 0 & 0.0237 \\
\hline 44 & 0.0467 & 0 & 0 & 0 \\
\hline 45 & 0 & 0 & 0 & 0.0348 \\
\hline 46 & 1.295 & 0.0114 & 0 & 0 \\
\hline $1-T_{\mathrm{av}} / T_{\mathrm{av} 0}$ & 0 & 0 & 0.6708 & 0.749 \\
\hline$m_{\mathrm{m}} / m_{\mathrm{m} 0}-1$ & 0 & 0.0315 & na & 0 \\
\hline
\end{tabular}

A similar analysis for other active constraints shows that an increase of the motor efficiency through a small modification of the feasible region is very little.

In general, the formula (9) can be used to estimate, without a recalculation of the problem, how the feasible region should be modified to improve the results. It is an advantage of applying a gradient routine over direct-search ones, including heuristic ones, e.g. genetic algorithms.

\section{Remarks concerning accuracy}

Due to a lack of a prototype manufactured on a base of the selected optimal design, the obtained results have been verified by means of FEM calculations. They have been performed in the paper before and after the optimization.

The electrical circuit of the converter in a form coupled with FEM model is presented on Figure 9.

Switches are controlled by means of the turn-off angle $\varphi_{\text {off }}$ used in the definition (3). In the analytical model the value $\varphi_{\text {off }}$ is determined to obtain the reference input active power $P_{\text {in }}=35 \mathrm{~kW}$. The same value of the $\varphi_{\text {off }}$ has also been assumed in the FEM model causing a difference in the $P_{\text {in }}$. According to this, the $T_{\text {av }}$ values in the FEM model were approximated linearly to meet the reference value of the $P_{\text {in }}$. Corrected results for the $T_{\text {av }}$ are presented in Table 5 and denoted as $T_{\mathrm{av}, \mathrm{FEM}}{ }^{*}$. 


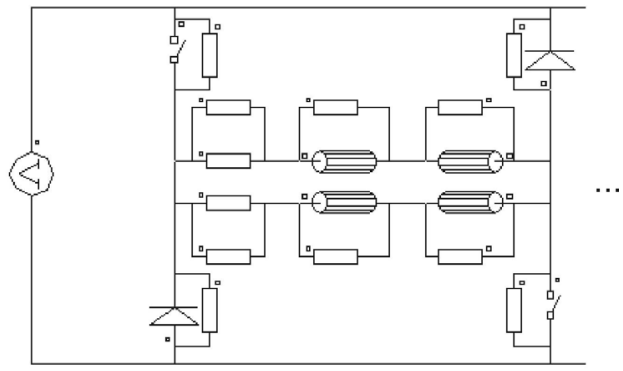

Fig. 9. A scheme of a one phase of electrical circuit coupled with the FEM model.

Shadowed elements represent phase winding sides

Table 5. A correction of $T_{\text {av }}$ values in FEM models

\begin{tabular}{c|c|c|c|c|c|c}
\hline \multirow{2}{*}{ Quantity } & Starting point & \multicolumn{5}{c}{ Problem } \\
\cline { 2 - 7 } & $\mathbf{x}_{0}$ & $\mathrm{P} 1$ & $\mathrm{P} 2$ & $\mathrm{P} 3$ & $\mathrm{P} 4$ & $\begin{array}{c}\text { P5 } \\
\text { (selected) }\end{array}$ \\
\hline$P_{\mathrm{n}, \mathrm{AM}}(\mathrm{kW})$ & \multicolumn{7}{|c}{35} \\
\hline$P_{\mathrm{n}, \mathrm{FEM}}(\mathrm{kW})$ & 36.17 & 34.24 & 34.93 & 36.02 & 34.80 & 35.56 \\
\hline$T_{\mathrm{av}, \mathrm{AM}}(\mathrm{Nm})$ & 88.20 & 89.59 & 89.55 & 88.23 & 88.20 & 90.00 \\
\hline$T_{\mathrm{av}, \mathrm{FEM}}(\mathrm{Nm})$ & 89.28 & 84.64 & 87.00 & 88.77 & 85.64 & 88.71 \\
\hline$T_{\mathrm{av}, \mathrm{FEM}}{ }^{*}(\mathrm{Nm})$ & 86.39 & 86.52 & 87.17 & 86.26 & 86.12 & 87.32 \\
\hline
\end{tabular}

Quantities $T_{\text {av2max }}$ and $\eta$ has not been approximated because they are relative. The noise level $L_{\mathrm{wA}}$ has been approximated in a more sophisticated way, due to the logarithmic function in its definition. The $L_{\mathrm{wA}}$ in FEM model was calculated with the help of the same algorithm as in the analytical model, but with the magnetic radial force $F_{\text {rad }}$ calculated in the FEM model.

\section{Remarks and conclusions}

The computer program used in the paper was implemented in Matlab environment with optimization routine based on Sequential Quadratic Programming (SQP), which appears to be particularly effective [24], [25], [9]. The time of calculations performed for one call of the synthesis program (determining all criterial and constraint functions) was below $60 \mathrm{sec}$ on a PC with Intel PIV 3.0 GHz processor.

The proposed approach has proved to be useful when searching for the best design of an SRM. An extension of the model by a better representation of a converter, considering the mutual inductances of the stator phase winding as well as heating model, would increase the credibility of optimization results. The described optimization procedure with analytical model and gradient routines has the following advantage over direct-search methods (e.g. heuristic ones) with FEM models: 
- Dramatically less calculation time by acceptable accuracy, particularly for higher number of optimization variables and complex constraint functions. A careful analysis and tests show that employing a FEM model instead of the analytical one would increase the calculation time even a few hundreds times, if the same optimization routine is employed. Applying commonly used heuristic optimization routines would increase this time even more because of a high convergence rate of the second order gradient algorithms like SQP used in the paper.

- More sophisticated optimization problems can be solved.

- Much more detailed analysis of obtained optimization results is possible, increasing the credibility of arising conclusions and decisions.

Model described in the paper requires the definition of an efficient algorithm for tube formation during optimization. Obtained results show that it is possible, but it is cumbersome and requires verification by means of FEM [14]. There are models comprising FEM calculation modules, which are active during the entire optimization period. An example would be the method and model CE-FEM (Finite Computationally Efficient-Element Analysis) described in [26]. In this method, the time-stepping FE calculations are replaced by magneto-static ones, thereby resulting in a significant reduction in computation time, even circa 100 times. The method was developed for the IPM motor, does not include the eddy currents and assumes that during the optimization functions of the currents in the stator windings are known and monoharmonic. Unfortunately, this assumption is not met in the case of SRM. In addition, the properties of $\mathrm{FE}$ calculations are the reason that the resulting criterial and constraint functions are not, in general, even the class $\mathrm{C}^{0}$. This prevents the use of gradient optimization algorithms in the approach represented by the CE-FEM type methods, until a way to circumvent this difficulty will be found. The use of the gradient method is advantageous for at least two reasons. Firstly, it enables performing sensitivity analysis in a vicinity of optimal point, as illustrated in Section 3.E of the paper for a solution of Problem P2. Secondly, in the case of multi-criteria optimization, every solution of the problem is Pareto-optimal, see Figure 7. The heuristic algorithms used in CE-FEM method must generate a much larger set of solutions, of which only a few can be considered as an estimate of the Pareto-optimal set [27]. Another important argument for the use of gradient methods is also the ability to validate the formal correctness of the model to a greater extent than the heuristic methods. Although construct a reliable model for gradient methods is more difficult than for the heuristic, this work is justified.

Drive with SRM requires a converter and controller, and in practical applications this should be included in the optimization. There are strong reasons to do this using a two-level optimization [28, Chapter 1]. In this approach, on the first level optimization is performed for discrete variables, while on the second one - for continuous part of the system. On the first level is changed, inter alia, the topology of the system and are defined constants used in the definitions of criterion functions and constraints at the second level (eg, peak current value and the dc link voltage of the inverter). In this paper we limited the procedure to the second level optimization because its purpose was only to demonstrate the effectiveness of the proposed model. There are no obstacles for a specific application, to extend the definition of feasible region on the second level with additional elements connecting the two levels of 
optimization. In such a case it is necessary decision on the choice of the vehicle, which usually has a significant impact on the topology of the motor, converter, and controller.

The power of the SRM at a fixed control decreases with increasing speed. For example, in the solution marked "selected" on Figure 7 input power was $P_{1}=35 \mathrm{~kW}$ for a base speed of $n=3500 \mathrm{rpm}$ and the assumed control $\varphi_{\text {on }}=0, \varphi_{\text {off }}=30^{\circ}$. After increasing the speed to $n=10000 \mathrm{rpm}$ and at the same one-pulse control settings, power has decreased to a value of approx. $8.5 \mathrm{~kW}$, ie approx. $25 \%$ of the previous. Of course angles $\varphi_{\text {on }}<0, \varphi_{\text {off }}>30^{\circ}$ (it is permissible for the speed $10000 \mathrm{rpm}$ ) would improve this result, but probably not enough to get a power of $35 \mathrm{~kW}$. The above calculations indicate that this should be taken into account in the optimization, since SRM are usually used in drives with a large range of variation of speed. The way how to do this depends on what is permissible power loss with increasing speed, and the vehicle, where the drive is to be installed. This can be an additional criterial function for the vector optimization, and in the case of a scalar optimization - scaled component of the objective function or a suitable penalty function, which is part of the objective function. The parameters of these functions are dependent on the particular application; their values are usually determined by trial and error. At the current stage of research, which relate to the model, this has not been included in the paper.

\section{Appendix}

The quantity $x_{\mathrm{i} 0}$ is an initial value of the $i$ th optimization variable $x_{\mathrm{i}}, i=1: 11$ (see Table 1). The other quantities are:

- $h_{\mathrm{s}}=x_{3}-x_{4}, h_{\mathrm{r}}=x_{4}-x_{9}-x_{7}, r_{\mathrm{rp}}=x_{4}-x_{9}$,

- $\alpha_{\mathrm{a}, \mathrm{b}, \ldots .}=\varphi_{\mathrm{a}, \mathrm{b}, \ldots . .}+\pi / N_{\mathrm{r}}$,

- $S_{\text {win }}, S_{\text {win, } \text { max }}$ - cross section area of the winding between stator poles, and its maximum value,

- $B_{\mathrm{msp}}, B_{\mathrm{msy}}, B_{\mathrm{mrp}}, B_{\mathrm{mry}}$ - maximum values of the flux density time waveforms in selected points of the magnetic core.

The feasible region is defined by constraints in Tables 6 and 7 .

Table 6. Inequality linear constraints

\begin{tabular}{c|c|c}
\hline No. & Constraint & Remarks \\
\hline $2-12$ & $x_{i} \geq 0.8 x_{i 0}, i=1: 11$ & $\operatorname{arbitrary}(*)$ \\
& $x_{6} \geq x_{60}, x_{9} \geq x_{90}$ & $\operatorname{arbitrary}(*)$ \\
\hline $13-23$ & $x_{i} \leq 1.2 x_{i 0}, i=1: 11$ & $\operatorname{arbitrary}(*)$ \\
\hline 24 & $x_{2} \leq x_{20}$ & $\operatorname{arbitrary}(*)$ \\
\hline 25 & $x_{3} \leq x_{2}-0.5 x_{1}$ & $\operatorname{arbitrary}(*)$ \\
\hline 26 & $x_{3} \geq 1.25 x_{4}$ & $\operatorname{arbitrary}(*)$ \\
\hline 27 & $r_{\mathrm{rp}} \geq 1.05 x_{7}$ & $\operatorname{arbitrary}(*)$ \\
\hline 28 & $x_{7} \geq 0.5 x_{1}+x_{6}$ & inductance calculations limitations \\
\hline 29 & $x_{7} \leq 0.75 x_{1}+x_{6}$ & \\
\hline
\end{tabular}




\begin{tabular}{c|c|c}
\hline 30 & $h_{\mathrm{s}} / h_{\mathrm{r}} \leq 2$ & inductance calculations limitations \\
\hline 31 & $1 / 2 \leq h_{\mathrm{s}} / h_{\mathrm{r}}$ & inductance calculations limitations \\
\hline 32 & $\left(x_{2}-x_{3}\right) / x_{1} \leq 0.7$ & according to [19] \\
\hline 33 & $2\left(x_{2}-x_{3}\right) /\left(x_{2}+x_{3}\right) \leq 0.2$ & $\begin{array}{l}\text { magnetic noise model limitations } \\
\text { (used only in noise optimization) }\end{array}$ \\
\hline 34 & $x_{8} /\left(x_{2}+x_{3}\right) \leq 1.1$ & $\begin{array}{l}\text { magnetic noise model limitations } \\
\text { (used only in noise optimization) }\end{array}$ \\
\hline
\end{tabular}

$(*)$ - the purpose of these constraints was to keep the optimal solutions, relatively close to the initial design, which was verified experimentally, in [16], [18]; it increases the credibility of final results

Table 7. Non-linear constraints

\begin{tabular}{|c|c|c|}
\hline No. & Constraint & Remarks \\
\hline 1 & $P_{\text {in }}=35000$ & requirement of rated power \\
\hline 35 & $\beta_{\mathrm{s}}<\beta_{\mathrm{r}}$ & \multirow{3}{*}{ according to $[23]$} \\
\hline 36 & $\beta_{\mathrm{s}} \geq 0.95 * 2 \pi /\left(N_{\mathrm{ph}} * N_{\mathrm{r}}\right)$ & \\
\hline 37 & $\beta_{\mathrm{s}}+\beta_{\mathrm{r}} \leq \pi / 2$ & \\
\hline 38 & $\alpha_{b} / \alpha_{a} \geq 1.04$ & \multirow{3}{*}{$\begin{array}{l}\text { a protection against inaccuracy and instability } \\
\text { of analytical solution of the Equation (2) }\end{array}$} \\
\hline 39 & $\alpha_{c} / \alpha_{a} \geq 1.27$ & \\
\hline 40 & $L\left(\varphi_{\mathrm{e}}\right)_{\text {nonsat }} / L\left(\varphi_{\mathrm{e}}\right)_{\text {sat }} \leq 9$ & \\
\hline 41 & $S_{\text {win }} \leq S_{\text {win, } \max }$ & a feasibility of the phase winding \\
\hline 42 & $B_{\mathrm{msp}} \leq 1.65$ & \multirow{4}{*}{$\begin{array}{l}\text { a limitation of flux density value to be below } \\
\text { the saturation region }\end{array}$} \\
\hline 43 & $B_{\mathrm{msy}} \leq 1.65$ & \\
\hline 44 & $B_{\mathrm{mrp}} \leq 1.65$ & \\
\hline 45 & $B_{\mathrm{mry}} \leq 1.65$ & \\
\hline 46 & $\varphi_{\text {off }} \leq 2 \pi /\left(N_{\mathrm{ph}} * N_{\mathrm{r}}\right)=\pi / 6$ & a protection against a mutual coupling \\
\hline
\end{tabular}

\section{References}

[1] Rahman K.M., Schulz S.E., Design of High-Efficiency and High-Torque-Density Switched Reluctance Motor for Vehicle Propulsion. IEEE Transactions on Industry Applications 38(6): 1500-1507 (2002).

[2] Crevecoeur G., Dupré L., Van de Walle R., Space Mapping Optimization of the Magnetic Circuit of Electrical Machines Including Local Material Degradation. IEEE Transactions on Magnetics 43(6): 2609-2611 (2007).

[3] Omekanda A.M., A New Technique for Multidimensional Performance Optimization of Switched Reluctance Motors for Vehicle Propulsion. IEEE Transactions on Industry Applications 39(3): 672676 (2003).

[4] Matveev A., Development of Methods, Algorithms and Software for Optimal Design of Switched Reluctance Drives. PhD thesis, Eindhoven University of Technology (2006).

[5] Anwar M.N., Husain I., Radun A.V., A Comprehensive Design Methodology for Switched Reluctance Machines. IEEE Transactions on Industry Applications 37(6): 1684-1692 (2001).

[6] Lukaniszyn M, Kowol M, Kołodziej J., Optimization of a two-phase transverse flux switched reluctance motor with an outer rotor. Archives of Electrical Engineering 61(4): 567-578 (2012).

[7] Kowol M., Łukaniszyn M., Latawiec K.J., Modelling and construction optimization of a modular TFM with an outer rotor. Electrical Engineering 92: 111-118 (2010). 
[8] Mirzaeian B., Moallem M., Tahani V., Lucas C. Multiobjective Optimization Method Based on a Genetic Algorithm for Switched Reluctance Motor Design. IEEE Transactions on Magnetics 38(3): 1524-1527 (2002).

[9] Choi C., Lee D., Park K., Fuzzy Design of a Switched Reluctance Motor Based on the Torque Profile Optimization. IEEE Transactions on Magnetics 36(5): 3548-3550 (2000).

[10] Majchrowicz M., Analytical Model of Switched Reluctance Motor for its Optimization. Elektrotechnika i Elektronika 26(1-2): 46-53 (2007) (in Polish).

[11] Jazdzynski W., Majchrowicz M., An Approach to Find an optimum Designed SRM for electric vehicle drive. Proceedings of the 2008 International Conference on Electrical Machines, Portugal, Vilamoura: Paper ID 1391 (2008).

[12] Majchrowicz M., Jażdżyński W., Selected aspects of an optimization of a switched reluctance (SRM) for an electric vehicle drive. Zeszyty Problemowe - Maszyny Elektryczne 84/2009: 147-150, XLV International Symposium on Electrical Machines, SME’2009, Poland, Rzeszów-Krasiczyn (2009) (in Polish).

[13] Majchrowicz M., Jazdzynski W. Selected Problems of Optimization of a Switched Reluctance Motor for an Electric Vehicle using Analytical Calculations. International Conference on Renewable Energies and Power Quality (ICREPQ'10), Spain, Granada: Paper ID 483 (2010).

[14] Majchrowicz M., Optimization of the Switched Reluctance Motor for an Electric Vehicle Drive. PhD thesis (in Polish), AGH University of Science and Technology (2011).

[15] Jazdzynski W., Majchrowicz M., Analytical Model of a Switched Reluctance Motor for its Optimization - Selected Problems. Electrical Review 89(11): 153-158 (2013).

[16] Bausch H., Greif A., Lange B., Bautz R. A $50 \mathrm{kW/15000} \mathrm{rpm} \mathrm{Switched} \mathrm{Reluctance} \mathrm{Drive} \mathrm{for} \mathrm{an}$ Electric Vehicle: Current Control and Performance Characteristics. Proceedings of XIV International Conference on Electrical Machines, Finland, Espoo: 603-607 (2000).

[17] Suzuki T., Ito S., Tanaka N. et al., Development of High-Efficiency Switched Reluctance Motor. Electrical Engineering in Japan 162(2): 73-82 (2008)

[18] Bausch H., Greif A., Nickel A.B.A., A Switched Reluctance and an Induction Machine in a Drivetrain for an Electrical Vehicle Under the Conditions of a Car Application, Proceedings of XIV International Conference on Electrical Machines, Finland, Espoo: 1313-1316 (2000).

[19] Krishnan R., Switched Reluctance Motor Drives: Modeling, Simulation, Analysis, Design, and Applications. (CRC Press LLC, 2001).

[20] Anwar M.N., Husain I., Radial Force Calculation and Acoustic Noise Prediction in Switched Reluctance Machines, IEEE Transactions on Industry Applications 36(6): 1589-1597 (2000).

[21] Gieras J.F., Wang C., Lai J.C., Noise of Polyphase Electric Motors. CRC Press, Taylor \& Francis Group (2006).

[22] Rasmussen P.O., Blaabjerg F., Pedersen J.K. et al., Acoustic noise simulation for Switched Reluctance Motors with audible output. Proceedings of European Conference on Power Electronics and Applications, Switzerland, Lausanne: 1-13 (1999).

[23] Miller T.J.E., Brushless Permanent-Magnet and Reluctance Motor Drives. Clarendon Press (1989).

[24] Schittkowski K., Nonlinear Programming Codes. Springer-Verlag (1980).

[25] Jazdzynski W., Multicriterial optimization of squirrel-cage induction motor design. IEE Proceedings 136 Pt. B(6): 299-307 (1989).

[26] Sizov G.Y., Ionel D.M., Demerdash N.A.O., Modeling and Parametric Design of Permanent-Magnet AC Machines Using Computationally Efficient Finite-Element Analysis. IEEE Transactions on Industrial Electronics 59(6): 2403-2413 (2012).

[27] Sizov G.Y., Peng Zhang, Ionel D.M. et al., Automated bi-objective design optimization of multi-MW direct-drive PM machines using CE-FEA and differential evolution. Energy Conversion Congress and Exposition (ECCE), IEEE: 3672-3678 (2011).

[28] Jażdżyński W., Designing electric machines and identification of their models using multi-criteria optimization. AGH University of Science and Technology, a series of "Dissertations and Monographs" 28, Kraków (1995) (in Polish). 